\title{
Head-Disk Interface Technology: Challenges and Approaches*
}

\begin{abstract}
Bo LIU**
Magnetic hard disk drive (HDD) technology is believed to be one of the most successful examples of modern mechatronics systems. The mechanical beauty of magnetic HDD includes simple but super high accuracy positioning head, positioning technology, high speed and stability spindle motor technology, and head-disk interface technology which keeps the millimeter sized slider flying over a disk surface at nanometer level slider-disk spacing. This paper addresses the challenges and possible approaches on how to further reduce the slider disk spacing whilst retaining the stability and robustness level of head-disk systems for future advanced magnetic disk drives.
\end{abstract}

Key Words: Mechatronics, Magnetic Hard Disk Drives, Magnetic Recording, Head-Disk Interface, Tribology

\section{Introduction}

Magnetic data storage technology, integrated circuit technology, and network technology are referred to as the 3 pillars for the infrastructure of the modern information technology. Also, information collection, information transferring and information storage are named as the 3 key aspects of our information age. Among them, information storage is of accumulation effect: people would like to store as much information collected/received as possible.

The technology representative of modern magnetic data storage devices and systems is the magnetic hard disk drive (HDD). Magnetic HDD includes a data storage disk which is mounted on a high speed spindle motor, a readwrite head which is carried by a slider-suspension system and flies over the disk surface at extremely small head-disk spacing, head positioning actuator and its servo electronics, and signal processing systems.

The magnetic HDD technology is one of the most successful nano-technology according to its technology level and market size. Mechanically, the head-disk spacing is at nanometer range $(7-10 \mathrm{~nm})$ now and the intermolecular interactions between slider and disk surface is becoming a big challenge in designing a stable and robust

\footnotetext{
* Received 14th March, 2005 (Review)

** Data Storage Institute, 5 Engineering Drive 1, 117 608, Singapore. E-mail: LIU_Bo@dsi.a-star.edu.sg
}

head-disk systems. Magnetically, the magnetic disk and head technology has reached such a level that nano-spin and electronics motion must be considered in both head and magnetic disk designs.

The magnetic HDD technology is one of the most successful mechatronics systems as well.

The functional performance of magnetic HDD is largely measured by achievable areal density over disk surface (Giga-bits per square inch). The latest achievement in areal density is $100 \mathrm{~Gb} / \mathrm{in}^{2}$ in production and $160 \mathrm{~Gb} / \mathrm{in}^{2}$ at lab level. Researchers are pushing technology innovation and development in all aspects so as to keep an annual areal density increase rate at $35-45 \%$.

This paper discusses challenges on head-disk interface technology and the possible solutions. The key parameter for head-disk interface is the average headdisk spacing and the stability of head disk spacing. Researchers are exploring technology solutions so as to moving the head-disk spacing from current level of $7-10 \mathrm{~nm}$ to merely $2.5-3.5 \mathrm{~nm}$ level or even contact recording.

\section{Challenges and Technical Approaches}

The areal density of present high end disk drives is around $100 \mathrm{Gbit} / \mathrm{in}^{2}$. Great efforts are being made to push technology towards $1000 \mathrm{Gbit} / \mathrm{in}^{2}$ or $1 \mathrm{Tbit} / \mathrm{in}^{2}$ (tera-bit per square inch) areal densities.

The suggested key parameters for $1 \mathrm{~Tb} / \mathrm{in}^{2}$ recording include a track density of $350-500 \mathrm{ktpi}$ (kilo tracks per 

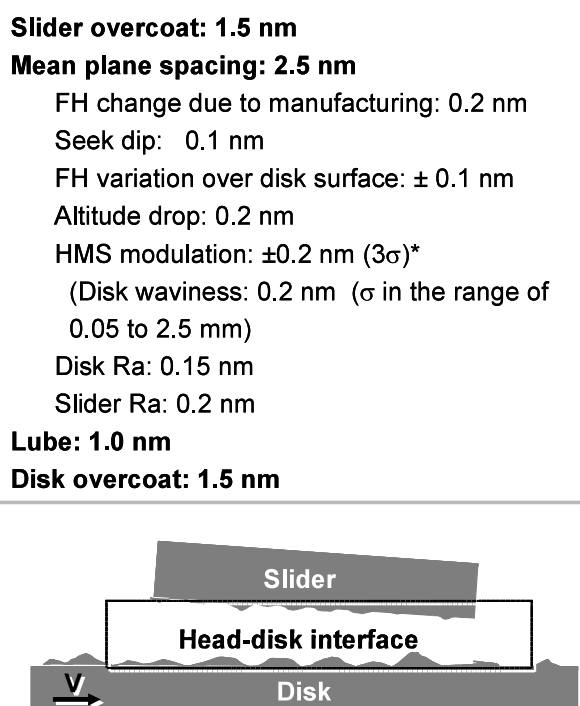

Fig. 1 Prediction of interface parameters with $2.5 \mathrm{~nm}$ head-disk spacing and for $\mathrm{Tb} / \mathrm{in}^{2}$ area density

inch), bit density of 2200-2800 kbpi (kilo bits per inch), grain diameter of $4.5-6 \mathrm{~nm}$, and merely $5.5-6.5 \mathrm{~nm}$ spacing between the read/write head and the recording media (head-media spacing) ${ }^{(1),(2)}$. Here, $5.5-6.5 \mathrm{~nm}$ magnetic spacing includes the following components: a mechanical head-disk spacing (the flying height) of $2.5-3.5 \mathrm{~nm}$, media protective layer thickness of $1-1.5 \mathrm{~nm}$, slider overcoat thickness of $1 \mathrm{~nm}$, and a lubricant thickness of $1 \mathrm{~nm}$. The recent success in the demonstration of $3.5 \mathrm{~nm}$ flying height slider technology ${ }^{(3)}$ leads the researchers to position the next flying height target at $2.5 \mathrm{~nm}$ or contact recording, aiming to accelerate the realization of $\mathrm{Tb} / \mathrm{in}^{2}$ areal densities.

Figure 1 schematically illustrates the prediction of parameters for achieving $2.5 \mathrm{~nm}$ mechanical spacing between head and disk surface. The parameters listed seem very difficult to achieve according to present practice of head-disk interface technology.

\subsection{Surface roughness and slider materials}

It can be seen that achieving super-smooth slider and disk surface are of great importance for such a head disk system. Current sliders are of a Ra value of $0.6 \mathrm{~nm}$ or above which is quite away from the expected $0.2 \mathrm{~nm}$. Furthermore, the current $\mathrm{Al}_{2} \mathrm{O}_{3} \mathrm{TiC}$ slider substrate composes of two types of grains: $\mathrm{Al}_{2} \mathrm{O}_{3}$ and TiC. Such two grains are of size at micron level and of different hardness and etching rate. It is not easy to polish them to the same height. In other words, it is not easy to significantly reduce the slider $\mathrm{Ra}$ value if $\mathrm{Al}_{2} \mathrm{O}_{3} \mathrm{TiC}$ is still being used as slider substrate.

Therefore, we need to explore new slider materials with achievable Ra value of $0.2-0.25 \mathrm{~nm}$, reasonable high electrical conductivity and high thermal conductivity.

\subsection{Disk surface roughness}

Disk surface roughness plays significant role when technology moves towards $3.5-2.5 \mathrm{~nm}$ mechanical spacing. Disk surface roughness comes from two aspects: intrinsic roughness which is the surface roughness of disk substrate (Al alloy or glass) and final surface roughness which is from the combined effect of intrinsic surface roughness and additional surface roughness caused by the deposition of thin-films (magnetic recording layer, intermediate layer, seed layer, and soft magnetic layer) for achieving good magnetic properties and recording performance of the disk media.

Magnetic recording technology is shifting from longitudinal magnetic recording scheme to perpendicular magnetic recording scheme. One significant difference between the two systems is that the total thickness of the magnetic recording related layers is significantly thicker in perpendicular magnetic recording case, comparing to that in the longitudinal magnetic recording case. In general, the thicker the sputtered layers, the greater the surface roughness increase.

For example, the intrinsic surface roughness of disk substrate can be $0.14 \mathrm{~nm}$. A layer of $46 \mathrm{~nm}$ thick soft magnetic underlayer will increase the surface roughness to $0.23 \mathrm{~nm}$, or an additional Ra increase of $0.09 \mathrm{~nm}$. A further $46 \mathrm{~nm}$ increase of the thickness of the same soft magnetic layer will further increase the Ra value to $0.27 \mathrm{~nm}$ or $0.04 \mathrm{~nm}$ further increase of surface roughness (AFM measurement over $5 \mu \mathrm{m} \times 5 \mu \mathrm{m}$ sized surface area).

The predicted disk Ra is only $0.15 \mathrm{~nm}$. Therefore, it is important to have glass substrate which is of small intrinsic surface roughness value. It is also important to improve the atomic level matching between different layers of materials. In fact, the different roughness increment between the first $46 \mathrm{~nm}$ thin-film thickness and the 2nd $46 \mathrm{~nm}$ thin film thickness indicate that material to material interface plays an important role in roughness change. Furthermore, it is preferred to make the thickness of the whole magnetic related layers as thin as possible, especially the soft magnetic underlayers and the intermediate layers between magnetic recording layer and the soft magnetic underlayer.

\subsection{Altitude induced flying height drop}

Disk drives need to work from sea level environment to mountain areas with altitude as high as $3000-4000 \mathrm{~m}$. Increased altitude also means reduced air molecule density and air pressure. As illustrated in Fig. 2, dropped air pressure will lead to the drop of both positive pressure (bearing force) and negative pressure (sucking force). In general, the net variation of positive force is larger than that of the negative force, if we could keep slider altitude fixed and evaluate the air bearing forces acting on slider body. As a result, the flying height of slider will be reduced.

For most sliders, an altitude increase from sea level 
to $3000 \mathrm{~m}$ will lead to a flying height drop of $20 \%$ around. However, the suggested budget for flying height loss caused by such altitude variation is $0.2 \mathrm{~nm}$ or only $8 \%$ of the nominal flying height. Therefore, it is important to come up with new schemes for air bearing force arrangement.

Figure 3 illustrates two sliders designed by author's team to work at $2.7 \mathrm{~nm}$ flying height. The sliders are of comparable pad layout. However, the force arrangement

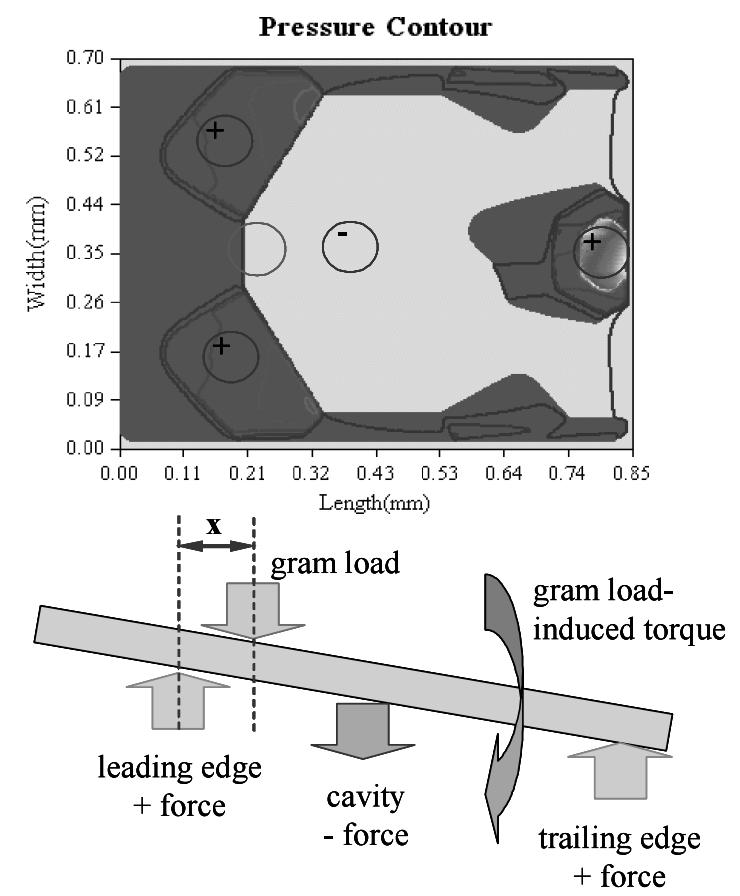

Fig. 2 Schematic illustration of schemes to reduce sensitivity of flying height to altitude variation
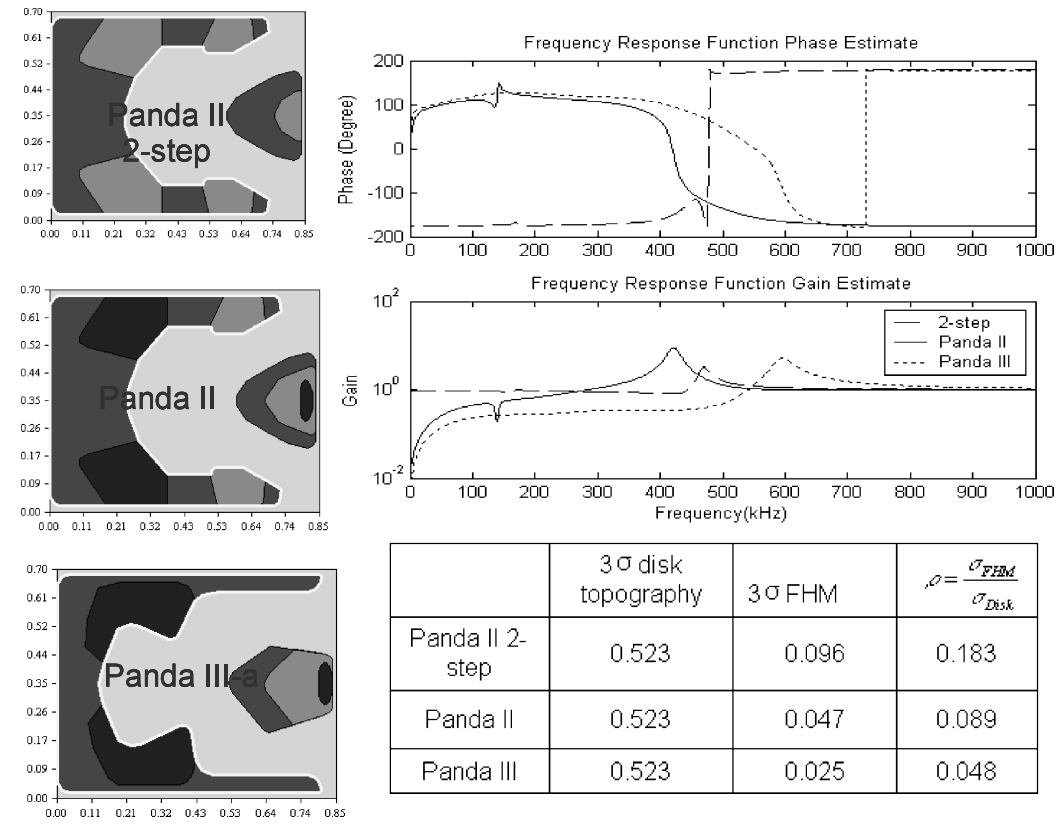

\begin{tabular}{|c|c|c|c|}
\hline & $\begin{array}{c}3 \sigma \text { disk } \\
\text { topography }\end{array}$ & $3 \sigma \mathrm{FHM}$ &,$\sigma=\frac{\sigma_{\text {FLMM }}}{\sigma_{\text {Disk }}}$ \\
\hline $\begin{array}{c}\text { Panda II 2- } \\
\text { step }\end{array}$ & 0.523 & 0.096 & 0.183 \\
\hline Panda II & 0.523 & 0.047 & 0.089 \\
\hline Panda III & 0.523 & 0.025 & 0.048 \\
\hline
\end{tabular}

Fig. 4 Reduction of flying height modulation strategy for the two types of sliders is different. Results suggest that the force arrangement for type (b) slider corresponds to a halved flying height drop when altitude increases from $0 \mathrm{~m}$ to $3000 \mathrm{~m}$, comparing with the type (a) slider.

\section{4 Slider's nano-deformation, disk surface wavi- ness and flying height modulation}

Authors $^{(4)}$ and other researchers reported that writing induced Joule heat will cause nano-deformation of slider body and reduced head disk spacing. Furthermore, disk is of surface waviness which will cause modulation of headdisk spacing as well.

Such nano-deformation and surface waviness can lead to a flying height drop up to $1-2 \mathrm{~nm}$ though the predicted budget for those effects is merely $0.3 \mathrm{~nm}$.

Thus, it is important to come up with proper schemes so as to significantly reduce the flying height modulation caused by such effects. The Panda II slider, proposed by author's team is illustrated in Fig. 4. As can be seen, the sub-shallow step concept introduced in Panda II slider leads to improved dynamic response of the slider and halved flying height modulation as well. Other than that, improved force arrangement in Panda III slider of the author's team further reduces the flying height modulation, as can be seen from the table listed in Fig. 4.
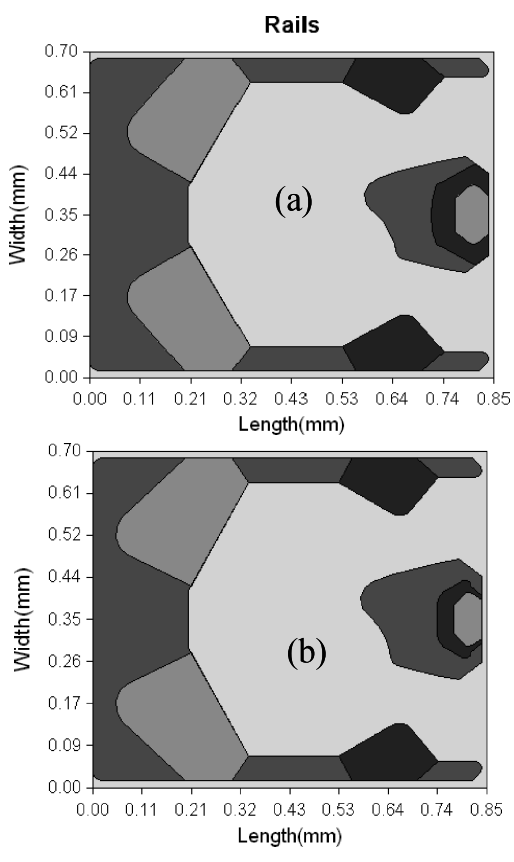

Fig. 3 Two types of slider designs. Type (a) is of an altitude sensitivity similar to most of the sliders used now and type (b) is of significantly reduced (halved) sensitivity to altitude variation. Both sliders are designed to work at $2.7 \mathrm{~nm} \mathrm{FH}$. 


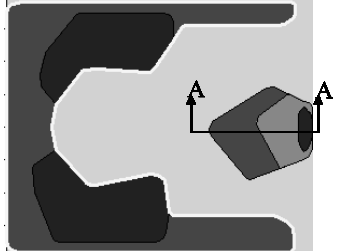

Slider ABS Layout

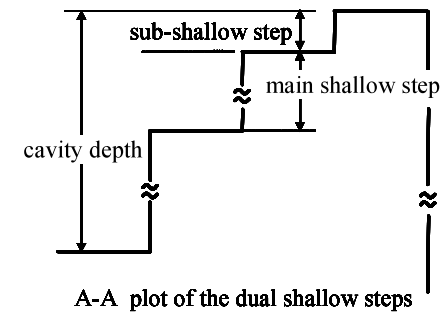

A-A plot of the dual shallow steps
Fig. 5 Dual shallow step arrangement

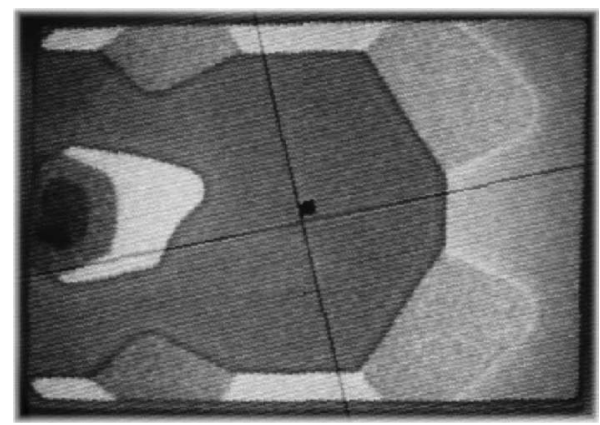

Fig. 6 Fabricated $2.7 \mathrm{~nm}$ FH slider under optical flying height tester

\subsection{High air pressure and high stiffness at trailing edge of slider body}

The author and his team noticed that increasing the concentration of air-pressure around read-write element will be of advantage to increased air-bearing stiffness, reduced sensitivity of flying height to disk waviness, reduced effect of Joule head induced slider deformation to flying height and increased heat transferring between $\mathrm{read} / \mathrm{write}$ head and disk surface.

For conventional sliders, the maximum air pressure at slider's trailing edge is around 10 times of ambient pressure. With the proposed sub-shallow step concept, as illustrated in Fig. 5, the air pressure can be largely concentrated to the small sub-shallow step pad and can achieve a maximum air pressure 18 times the ambient pressure (same gram load of $0.8 \mathrm{~g}$ ). Optimized shallow step slider in author's team can achieve very highly concentrated air pressure and a maximum air-pressure up to 70 times of the ambient pressure, with same suspension as used by others.

\section{Acknowledgment}

The author would like to thank the team members, including Mr. Leonard Gonzaga, Ms. Jin Liu, Dr. Shengkai Yu, Mr. Yufei Han, Dr. Mingsheng Zhang, Mr. Jiang Zhou, Mr. Hui Li, for the design, fabrication and testing of the sliders mentioned in this paper.

\section{References}

( 1 ) Wood, R., The Feasibility of Magnetic Recording at $1 \mathrm{~Tb} / \mathrm{in}^{2}$, IEEE Trans. Magn., Vol.36 (2000), pp.36-42.

(2) Liu, B., Liu, J. and Chong, T.-C., Slider Design for Sub-3 nm Flying Height Head-Disk Systems, J. Magn. Magn. Mater., Vol.287 (2005), pp.339-345.

( 3 ) Liu, B., Liu, J., Jianfeng et al., Flying Height Modulation and Femto Slider Design with $3.5 \mathrm{~nm}$ Flying Height, the 4th International Conference on Tribology of Information Storage Devices, Dec., Monterey, USA, (2003).

(4) Liu, B. and Han, Y.F., Writing Induced Crown Change and Its Effect to Head-Disk Clearance, J. Appl. Phys., Vol.93, No.10 (2003), pp. 8716-8718.

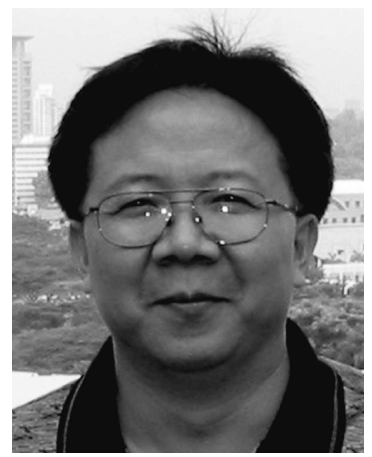

Bo Liu

Bo LIU received his Ph.D degrees in Electrical Engineering from the University of Manchester, Manchester, England, in 1993 and his B.Sc and M.Eng from the Huazhong University of Science and Technology, Wuhan, P.R.China in 1982 and 1984, respectively. He has been working in the area of magnetic and magneto-optic recording field since 1984. He was with China's National Key Laboratory for Computer Peripheral Storage System in 1984-1990, working on magnetic recording theory, channel, disk drive and testing equipment development for perpendicular magnetic recording technology. He moved to the Information Storage Group of the Manchester University, Manchester, England, for his Ph.D study, working magnetooptical and magnetic recording. He jointed the Data Storage Institute (DSI), Singapore, in 1993. At his latter establishment, he is the head of the Spintronics, Media and Interfaces Division of the Institute. His current research activities include low flying height head-disk interface technology, nano-metrology and instrumentation. He is member of IEEE and has more than 100 publications and 10 patens in the data storage field. 